University of Wollongong

Research Online

Faculty of Engineering and Information

Faculty of Engineering and Information

Sciences - Papers: Part A

Sciences

$1-1-2005$

Development and characterization of a high-resolution microSPECT system for small-animal imaging

Yujin Qi

Johns Hopkins Medical Institutions, yujin@uow.edu.au

Benjamin Tsui

Johns Hopkins Medical Institutions, btsui@jhmi.edu

Yuchuan Wang

Johns Hopkins Medical Institutions

Brian Yoder

Johns Hopkins Medical Institutions

R Wojcik

Thomas Jefferson National Accelerator Facility

See next page for additional authors

Follow this and additional works at: https://ro.uow.edu.au/eispapers

Part of the Engineering Commons, and the Science and Technology Studies Commons

Research Online is the open access institutional repository for the University of Wollongong. For further information contact the UOW Library: research-pubs@uow.edu.au 


\title{
Development and characterization of a high-resolution microSPECT system for small-animal imaging
}

\begin{abstract}
We developed a high-resolution microSPECT system and investigated its performance characteristics for small animal imaging. The microSPECT system consists of a stationary compact gamma camera with interchangeable pinhole and parallel-hole collimators and vertical object rotation mechanism. The modular camera is based on a pixellated $\mathrm{Nal}(\mathrm{TI})$ crystal array with $1.2 \mathrm{~mm}$ pixel size and $1.4 \mathrm{~mm}$ pixel pitch coupled to a 5" diameter Hamamatsu R3292 PSPMT. The pinhole collimator has a $10 \mathrm{~cm}$ focal length fitted with keel-edge pinhole apertures with diameters of $1.0,1.5$ and $2.0 \mathrm{~mm}$. The system performance was characterized by the measurements of the point source response function (PSRF) using $\sim 300 \mu \mathrm{m}$ point sources of $99 \mathrm{mTc}$. The system performance in SPECT imaging was evaluated using micro SPECT phantom and 99mTc-MDP bone scan of a mouse. The asymmetric PSRF and detectionefficiency falloff were observed for the pinhole apertures with keel-edge design when the point source was scanned from the central axis to the edge of the field-of-view of pinhole collimator. This system can achieve 1.1 $\mathrm{mm}$ spatial resolution using $1 \mathrm{~mm}$ pinhole aperture at $2.5 \mathrm{~cm}$ distance to the pinhole. High-resolution and high-quality SPECT images were demonstrated from imaging micro SPECT phantoms and 99mTc-MDP bone scan of a mouse.
\end{abstract}

\section{Keywords}

high, characterization, resolution, development, microspect, imaging, system, small, animal

\author{
Disciplines \\ Engineering | Science and Technology Studies
}

\section{Publication Details}

Qi, Y., Tsui, B., Wang, Y., Yoder, B., Wojcik, R., Majewski, S. \& Weisenberger, A. (2005). Development and characterization of a high-resolution microSPECT system for small-animal imaging. In M. A. Kupinski \& $H$. H. Barrett (Eds.), Small-animal spect imaging (pp. 259-266). Boston, MA: Springer Science+Business Media, Inc.

\section{Authors}

Yujin Qi, Benjamin Tsui, Yuchuan Wang, Brian Yoder, R Wojcik, S Majewski, and A G Weisenberger 


\title{
Chapter 21
}

\section{DEVELOPMENT AND CHARACTERIZATION OF A HIGH-RESOLUTION MICROSPECT SYSTEM FOR SMALL ANIMAL IMAGING}

\author{
Yujin Qi ${ }^{1}$, Benjamin M.W. Tsui ${ }^{1}$, Yuchuan Wang ${ }^{1}$, Bryan Yoder $^{1}$, \\ Randolph Wojcik ${ }^{2}$, Stan Majewski ${ }^{2}$ and Andrew G. Weisenberger ${ }^{2}$ \\ ${ }^{1}$ Division of Medical Imaging Physics, Department of Radiology, Johns Hopkins Medical \\ Institutions, Baltimore, MD 21287-0859, USA. ${ }^{2}$ Detector Group, Thomas Jefferson National \\ Accelerator Facility, Newport News, VA 23606, USA
}

\begin{abstract}
We developed a high-resolution microSPECT system and investigated its performance characteristics for small animal imaging. The microSPECT system consists of a stationary compact gamma camera with interchangeable pinhole and parallel-hole collimators and vertical object rotation mechanism. The modular camera is based on a pixellated $\mathrm{NaI}(\mathrm{Tl})$ crystal array with $1.2 \mathrm{~mm}$ pixel size and $1.4 \mathrm{~mm}$ pixel pitch coupled to a 5" diameter Hamamatsu R3292 PSPMT. The pinhole collimator has a $10 \mathrm{~cm}$ focal length fitted with keel-edge pinhole apertures with diameters of $1.0,1.5$ and $2.0 \mathrm{~mm}$. The system performance was characterized by the measurements of the point source response function (PSRF) using $\sim 300 \mu \mathrm{m}$ point sources of ${ }^{99 \mathrm{~m}} \mathrm{Tc}$. The system performance in SPECT imaging was evaluated using micro SPECT phantom and ${ }^{99 \mathrm{~m}} \mathrm{Tc}-\mathrm{MDP}$ bone scan of a mouse. The asymmetric PSRF and detectionefficiency falloff were observed for the pinhole apertures with keel-edge design when the point source was scanned from the central axis to the edge of the field-of-view of pinhole collimator. This system can achieve $1.1 \mathrm{~mm}$ spatial resolution using $1 \mathrm{~mm}$ pinhole aperture at $2.5 \mathrm{~cm}$ distance to the pinhole. High-resolution and high-quality SPECT images were demonstrated from imaging micro SPECT phantoms and ${ }^{99 \mathrm{~m}} \mathrm{Tc}-\mathrm{MDP}$ bone scan of a mouse.
\end{abstract}

Key words: microSPECT system, small animal imaging.

\section{INTRODUCTION}

The rapid growth in molecular genetics and the development of mouse models of human diseases have led to significant interest in in vivo small animal imaging. Several instrumentation approaches [1-4] have been utilized 
to develop high-resolution animal SPECT for functional small animal imaging. Compact modular cameras based on pixellated crystal and position sensitive photomultiplier tube (PSPMT) are a low cost alternative to conventional gamma cameras for use in pinhole SPECT systems with high detection efficiency and resolution [5]. We have developed a compact, highresolution microSPECT system in our lab for small animal imaging. Our approach was to use a dedicated mini camera with pinhole collimator. The modular camera was based on a pixellated $\mathrm{NaI}(\mathrm{Tl})$ crystal array and a single 5" Hamamatsu PSPMT. The system performance was characterized using small point source. The system spatial resolution and sensitivity were measured. High-resolution phantom and in vivo mouse imaging were performed to demonstrate the system capability.

\section{IMAGING SYSTEM AND METHOD}

Figure 23-1 shows the microSPECT system developed in our lab. The system was designed to have a simple motion control scheme where the camera was placed in horizontal stationary position while imaging object was rotated vertically in front of the collimator. The rotary stage was mounted on $(\mathrm{x}, \mathrm{y}, \mathrm{z})$ linear translation stage from the Velmex Inc. for accurately three-dimensional positioning of the object.

\subsection{Compact Gamma Camera}

The compact gamma camera is based on a pixellatted $\mathrm{NaI}(\mathrm{Tl})$ scintillation crystal array from Saint Gobain Crystals and Detectors Inc. attached to a 5" diameter Hamamatsu R3292 position sensitive PMT (PSPMT). The $11.2 \mathrm{~cm} \times 11.2 \mathrm{~cm}$ pixellated crystal array consists of $1.2 \times 1.2 \times 6 \mathrm{~mm}^{3}$ pixel elements separated by $0.2 \mathrm{~mm}$ gaps that are filled with optically opaque and reflective materials. The light output from the scintillator is detected by the optically coupled PSPMT using a subtractive resistive readout [6] to lead out four signal outputs

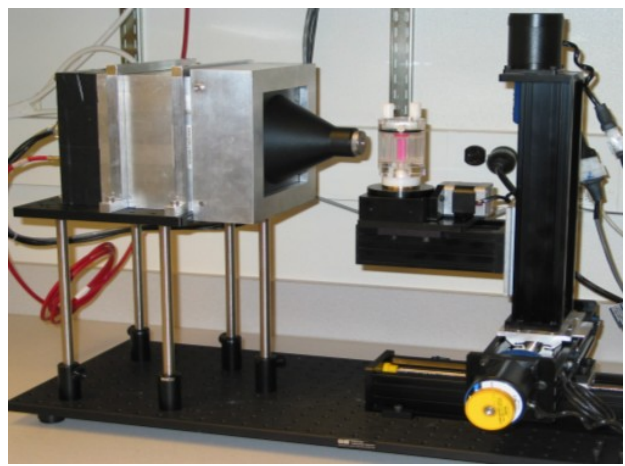

Figure 23-1. A photograph of the microSPECT system based on a compact gamma camera using a pixellated $\mathrm{Na}(\mathrm{Tl})$ crystal array and a 5" Hamamatsu R3292 PSPMT. The camera was placed in stationary position and the object was rotated vertically in front of the collimator. 
$\left(\mathrm{X}^{+}, \mathrm{X}^{-}, \mathrm{Y}^{+}\right.$and $\left.\mathrm{Y}^{-}\right)$. The signals were digitized by 12-bit PCI-6110E ADC card from the National Instruments. The data acquisition system was based on a Macintosh G3 workstation using the Kmax software package from the Sparrow Inc. A truncated centroid algorithm [7] was used to calculate the position and energy of the detected gamma photons. The effective field-ofview of the camera was $\sim 10.2 \mathrm{~cm}$ in diameter within a useful pixel array of $73 \times 73$. The camera was optimized for ${ }^{99 \mathrm{~m}} \mathrm{Tc}$ imaging. The measured energy resolution was $\sim 16 \% \mathrm{FWHM}$ for $140-\mathrm{KeV}$ photons. The energy window was set at $130-155 \mathrm{KeV}$ for ${ }^{99 \mathrm{~m}} \mathrm{Tc}$ imaging.

The camera can be fitted with interchangeable pinhole and parallel-hole collimators. The parallel-hole collimator designed for imaging the whole body of small animals and the pinhole collimator was used in imaging regional activity uptakes in the animals. The pinhole collimator has a focal length of $\sim 10 \mathrm{~cm}$ and an acceptance angle of $62^{\circ}$. The pinhole apertures were interchangeable and were made of tungsten alloy with diameters of 1.0, 1.5 and $2.0 \mathrm{~mm}$. The pinhole apertures had keel edge design to minimize the photon penetration. Two general-purpose parallel-hole collimators were designed for the camera, one for high-resolution and the other for highsensitivity imaging. The design parameters of the two parallel-hole collimators are listed in Table 23-1. The design of all the collimators was optimized for $140-\mathrm{KeV}$ photons.

The system was carefully calibrated for the system misalignments using a novel calibration method developed in our group [8]. The measured distance from the pinhole aperture to the image plane of the detector was $10.8 \mathrm{~cm}$.

Table 23-1. Design parameters of the two parallel-hole collimators

\begin{tabular}{cccccc}
\hline Type & Material & Technique & Hole length $(\mathrm{mm})$ & Hole size $(\mathrm{mm})$ & Septa $(\mathrm{mm})$ \\
\hline HRES & Lead & Cast & 40 & 1.4 & 0.16 \\
HSEN & Lead & Foil & 23 & 1.5 & 0.20 \\
\hline
\end{tabular}

\subsection{Experimental Method}

The performance of the microSPECT system was carefully characterized by its point source response function (PSRF) at different source positions. The full-width-at-half-maximum (FWHM) of the PSRF was used to determine the system spatial resolution while the volume under the PSRF was used to determine the system sensitivity.

The point sources used in the PSRF measurements were made with $\sim 300$ $\mu \mathrm{m}$ resin beads that absorbed high ${ }^{99 \mathrm{~m}} \mathrm{Tc}$ concentrations up to $0.1-0.2 \mathrm{mCi}$. We scanned the point source along the central axis and transaxially across the field-of-view (FOV) of the pinhole collimator. For each source position, a 10 minute planar image of the point source was acquired. From the 
measured PSRF, the system spatial resolution and sensitivity were determined as a function of source-to-collimator distance, source position offset from the central axis and the pinhole diameter.

The system performance in pinhole SPECT imaging was evaluated using a micro SPECT phantom from the Data Spectrum Inc. This cylindrical phantom has two types of inserts of $4.5 \mathrm{~cm}$ in diameter. The first insert is made with six sets of small plastic rods while the second insert has the same sets of hole channels. The rod and hole diameters are 1.2, 1.6, 2.4, 3.2, 4.0 and $4.8 \mathrm{~mm}$, respectively. The phantoms with either cold-rods or hotchannels were imaged using the $1.0 \mathrm{~mm}$ pinhole aperture. The micro SPECT phantoms were filled with $\sim 5 \mathrm{mCi}$ of ${ }^{99 \mathrm{~m}} \mathrm{Tc}$-pertechnetate in water solution. To avoid truncation in the projection data, the phantoms was placed at a distance of $\sim 5 \mathrm{~cm}$ from the pinhole aperture resulting in a magnification factor of $\sim 2$.

The projection data were acquired using 120 angular views equally spaced over $360^{\circ}$ and the acquisition time was 40 seconds per view. The radius-of-rotation (ROR) was $\sim 5 \mathrm{~cm}$, resulting in a magnification of $\sim 2$. The projection data were reconstructed using a 3D pinhole OS-EM algorithm with 10 iterations and 10 subsets. Reconstruction matrix size was $80 \times 80 \times 80$ with a voxel size of $0.35 \mathrm{~mm}$. A 3D Butterworth filter with cutoff frequency of 0.25 cycles/voxel was used for the post-reconstruction.

A mouse bone scan with ${ }^{99 \mathrm{~m}} \mathrm{Tc}-\mathrm{MDP}$ injection was also performed using $1.0 \mathrm{~mm}$ pinhole aperture to evaluate the pinhole micorSPECT image quality. The mouse was IV injected with $10 \mathrm{mCi}{ }^{99 \mathrm{~m}} \mathrm{Tc}-\mathrm{MDP}$ through the tail vein. The mouse was sacrificed and scanned after 2.5 hours postinjection. A total of 120 projections with angular views equally spaced over $360^{\circ}$ were acquired from the animal. The scan interval was 40 seconds per view. The ROR was $\sim 2.6 \mathrm{~cm}$, resulting in a magnification of $\sim 4$. The projection data were reconstructed using a 3D pinhole OS-EM algorithm with 6 iterations and 10 subsets and with correction of system geometric misalignments. Reconstruction matrix size was $80 \times 80 \times 80$ with a voxel size of $0.35 \mathrm{~mm}$. A 3D Butterworth filter with cutoff frequency of 0.3 cycle/voxel was used for the post-reconstruction.

\section{RESULTS}

The measured point source response functions for the keel-edge pinhole aperture using ${ }^{99 \mathrm{~m}} \mathrm{Tc}$ point source are shown in Figure 23-2. In the central axis direction, the image of the point source grows larger as the point source moves closer to the pinhole due to the magnification nature of the pinhole collimation. However, in the transaxial direction across the FOV of the 


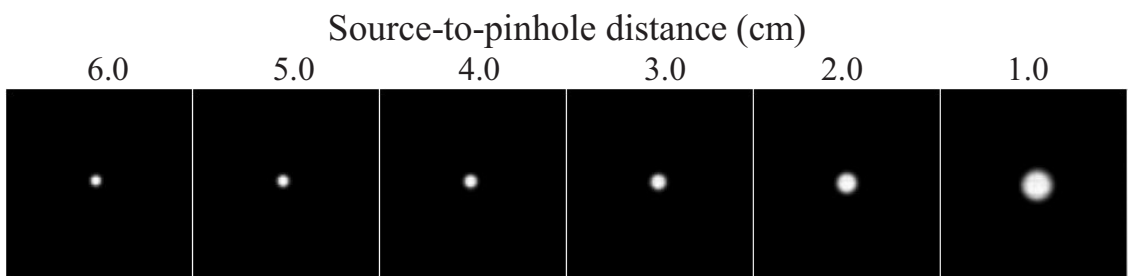

(a)

Source offset from the central axis $(\mathrm{mm})$

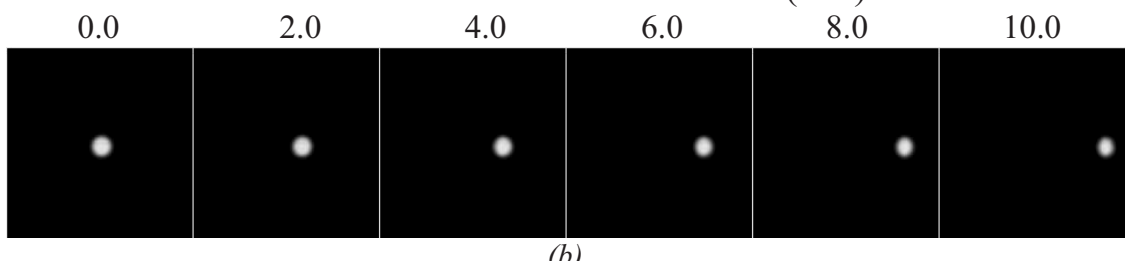

(b)

Figure 23-2. Sample of point source response function results obtained using a 300um diameter resin bead soaked with $0.15 \mathrm{mCi}{ }^{99 m} \mathrm{Tc}$ and $2.0 \mathrm{~mm}$ pinhole aperture with keeledge design: (a) along the axis direction and (b) along the transaxial direction at the source-to-pinhole distance of $2.5 \mathrm{~cm}$.

pinhole collimator, the PSRF becomes increasingly asymmetric as the point source moves further off the central axis of the pinhole collimator. The shape of the PSRF is narrower in the radial than in the tangential direction.

Figure 23-3 shows the axial spatial resolution and sensitivity as a function of source-to-collimator distance for the three pinhole apertures and two parallel-hole collimators. All three pinhole apertures provide much improved spatial resolution as compared to the parallel-hole collimators. In contrast with the parallel-hole collimators, the pinhole collimation exhibits a rapid increase in detection efficiency as the source-to-collimator distance

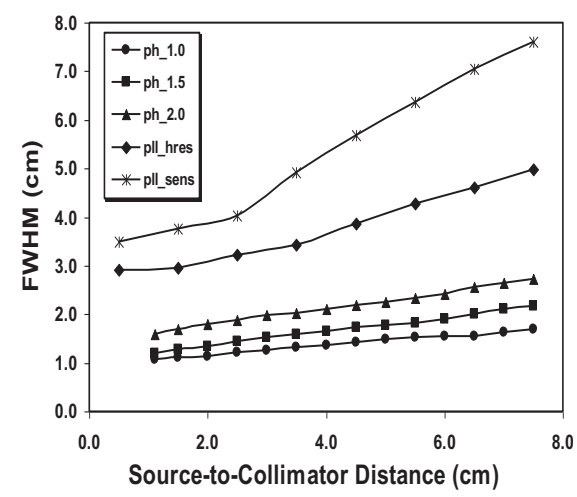

(a)

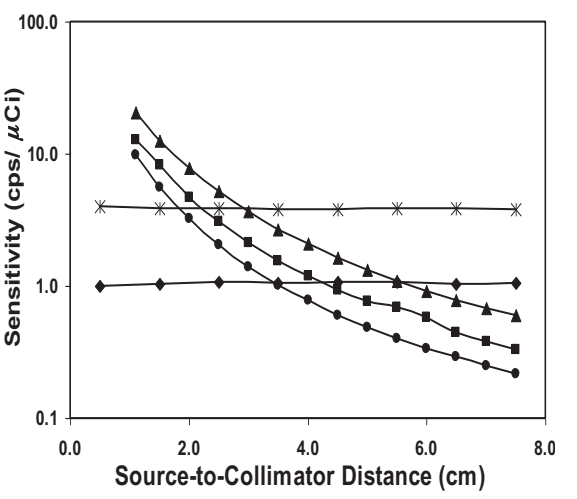

(b)

Figure 23-3. Measured axial (a) spatial resolution and (b) sensitivity of the microSPECT system as a function of source-to-collimator distance. 
decreases. However, the useful field-of-view is reduced as the distance is decreased. When imaging a mouse with a diameter of $2.5 \mathrm{~cm}$, we can place the center of the mouse as close as $\sim 2.6 \mathrm{~cm}$ from the pinhole aperture. Our system can achieve a spatial resolution and sensitivity of $\sim 1.2 \mathrm{~mm}$ and $\sim 2$ $\mathrm{cps} / \mu \mathrm{Ci}$ using the $1 \mathrm{~mm}$ pinhole aperture.

The transaxial spatial resolution and sensitivity for the three pinhole apertures are shown in Figure 23-4. The measurements were made at a source-to-pinhole distance of $2.5 \mathrm{~cm}$ using a $300 \mu \mathrm{m}$ diameter point source with $0.15 \mathrm{mCi}{ }^{99 \mathrm{~m}} \mathrm{Tc}$. The detection efficiencies for the three pinhole apertures show a significant falloff from the central axis to the edge of the FOV of the pinhole collimator. The PSRF shows increased asymmetry as the point source moves off the central axis to the edge of FOV of pinhole collimator with the a smaller FWHM values in the radial than in the tangential direction.

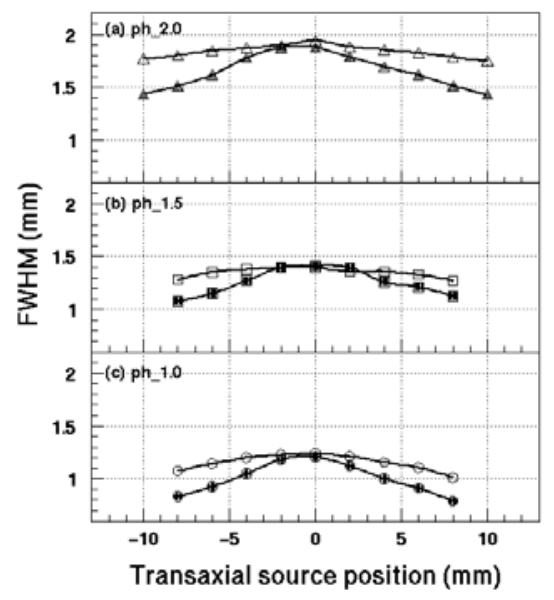

(a)

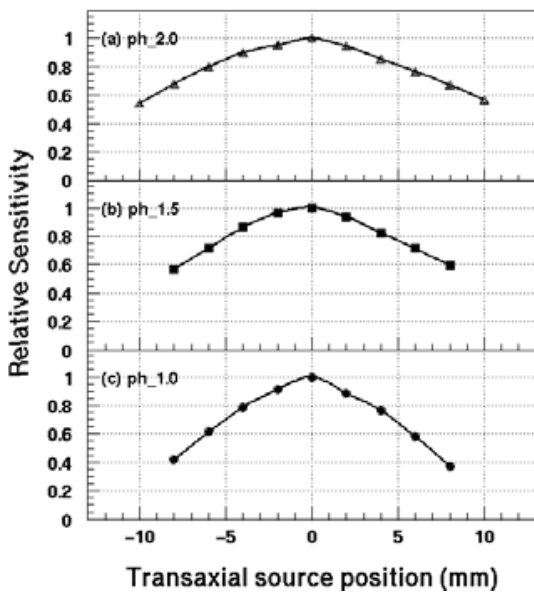

(b)

Figure 23-4: Measured (a) spatial resolution and (b) sensitivity of the microSPECT system in the transaxial direction for three pinhole apertures at source-to-pinhole distance of 2.5 $\mathrm{cm}$. In (a), the solid symbols denote the FWHM values in the radial direction while the open symbols represent the FWHM values in the tangential direction in the image plane.

Figure 23-5 shows the reconstructed transaxial images of the micro SPECT phantoms with cold-rods and hot-channels. With the $\sim 5 \mathrm{~cm}$ ROR and $\sim 2$ magnification, the microSPECT system with $1 \mathrm{~mm}$ pinhole aperture can clearly resolve both cold-rods and hot-channels from $4.8 \mathrm{~mm}$ down to $1.6 \mathrm{~mm}$ but not the $1.2 \mathrm{~mm}$ rods and hot-channels.

Figure 23-6 shows the reconstructed coronal images through the chest of a normal mouse. Individual ribs of the mouse are clearly resolved in the highresolution reconstructed images. The results demonstrate that our 
microSPECT System for Small Animal Imaging

microSPECT system based on compact camera is capable of high quality small animal imaging.

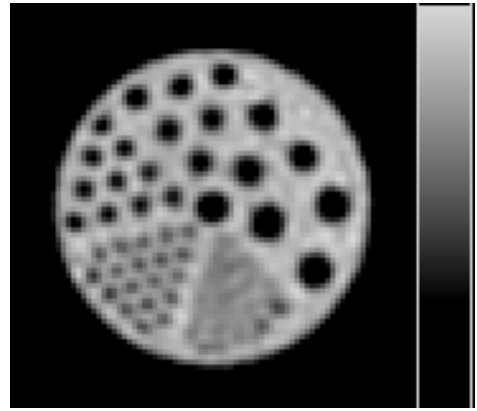

(a)

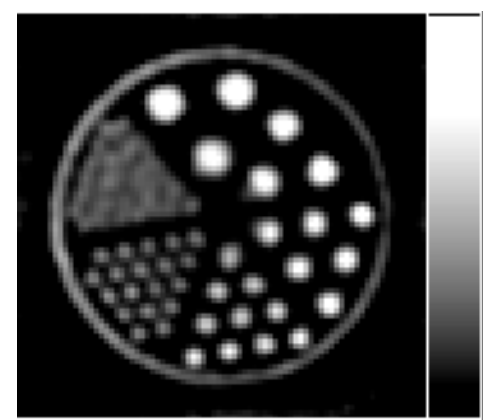

(b)

Figure 23-5. Reconstructed transaxial images of the micro SPECT phantoms with (a) coldrods and (b) hot-channels obtained from the microSPECT system using the $1.0 \mathrm{~mm}$ pinhole aperture. The radius-of-rotation was $\sim 5 \mathrm{~cm}$ in both cases. The phantom was filled with $\sim 5 \mathrm{mCi} 99 \mathrm{mTc}$-pertechnetate in water solution. The rod and hole diameters in the six sectors are 1.2, 1.6, 2.4, 3.2, 4.0 and $4.8 \mathrm{~mm}$.

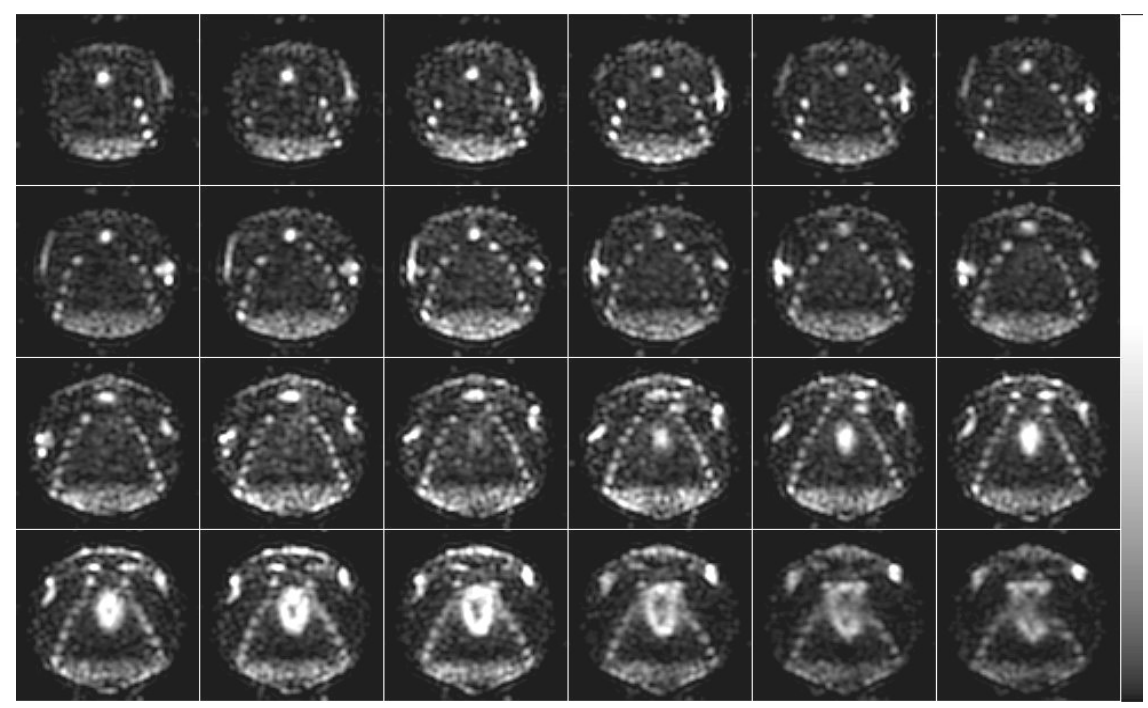

Figure 23-6. Pinhole SPECT bone scan of a normal mouse: coronal image slices through the chest of the mouse.

\section{DISCUSSION}

We have characterized the performance of a high-resolution microSPECT system based on a compact gamma camera with a pixellated 
crystal array and a 5" Hamamatsu PSPMT. As shown in the micro SPECT phantom images using ${ }^{99 \mathrm{~m}} \mathrm{Tc}$, our microSPECT system with pinhole collimator can clearly resolve the $1.6 \mathrm{~mm}$ cold-rods and hot-channels with a ROR of $\sim 5 \mathrm{~cm}$. When imaging a smaller object such as a mouse using a smaller ROR and larger magnification factor, improved resolution in order of 1-1.5 mm can be achieved. The measured point source sensitivities at 2.5 $\mathrm{cm}$ from the pinhole are approximately 2,3 and 5.2 counts $/ \mathrm{sec} / \mu \mathrm{Ci}$ for the $1.0,1.5$ and $2.0 \mathrm{~mm}$ pinhole apertures, respectively.

The PSRF of a pinhole collimator with keel-edge pinhole apertures becomes increasingly asymmetric towards the edge of the field-of-view of the collimator with concurrent decrease in sensitivity. The variations in PSRF and sensitivity are dependent on the aperture design parameters, including hole diameter and keel thickness, and position of the activity source relative to the pinhole aperture. These factors are important considerations in the specifications and design of a pinhole collimator for high-resolution SPECT imaging.

\section{CONCLUSION}

The modular camera based on a pixellated crystal array and a single 5" Hamamatsu PSPMT is an effective approach to high-resolution microSPECT system for small animal imaging. However, to achieve highresolution SPECT images, careful collimator design, system calibration and iterative image reconstruction methods with accurate correction of system geometric misalignments are required.

\section{ACKNOWLEDGEMENTS}

This investigation was supported by a startup fund from the Department of Radiology of the Johns Hopkins Medical Institutions. The authors thank Mrs. Ruth Hagemann for her assistance in the small animal experiments. Ray Visions Inc. provided the electronic circuitry for the resistive readout on the PSPMT. R. Wojcik, S. Majewski and A.G. Weisenberger would like to acknowledge the partial support from the Office of Biological and Environmental Research of the Office of Science of the U.S. Department of Energy. 


\section{REFERENCES}

[1] D.A. Weber, M. Ivanovic, D. Franceschi, S-E. Strand, K. Erlandsson, M. Franceschi, H.L. Atkins, J.A. Coderre, H. Susskind, T. Button and K. Ljunggren, "Pinhole SPECT: An approach to in vivo high resolution SPECT imaging in small laboratory animals," $J$. Nucl. Med., vol. 35, no. 2, pp. 342-348, February, 1994.

[2] B.M.W. Tsui, Y. Wang, E.C. Frey, and D.E. Wessell, "Application of ultra highresolution pinhole SPECT system based on a conventional camera for small animal imaging," J. Nucl. Med., vol. 42, no. 5, pp. 54P-55P, 2001.

[3] D.P. McElroy, L.R. MacDonald, F.J. Beekman, Y. Wang, B.E. Patt, J.S. Iwanczyk, B.M.W. Tsui, and E.J. Hoffman, "Performance evaluation of A-SPECT: A highresolution desktop pinhole SPECT system for imaging small animals," IEEE Trans. Nucl. Sci., vol. 49, no. 5, pp. 2139-2147, 2002.

[4] T.E. Peterson, H. Kim, M.J. Crawford, B.M. Cershman, W.C.J. Hunter, H.B. Barber, L.R. Furenlid, D.W. Wilson, J.M. Woolfenden, and H.H. Barratte, "SemiSPECT: A small-animal imaging system based on eight CdZnTe pixel detectors," 2002 IEEE NSSMIC Conference Record, Norfolk, Nov.10-16, 2002.

[5] Y.J. Qi, B.M.W. Tsui, Y. Wang, B. Yoder, R. Wojcik and S. Majewski, "Performance characteristics of a new modular camera based on pixellated crystal for high-resolution pinhole SPECT with comparison to a standard camera with continuous crystal," Conference Record: M7-60, IEEE Nuclear Science Symposium, 2003.

[6] R. Wojcik, S. Majewski, B. Kross, V. Popov, and A.G. Weisenberger, "Optimized readout of small gamma cameras for high resolution single gamma and positron emission imaging,"2001 IEEE NSS-MIC Conference Record, San Diego, Nov.4-10, 2001.

[7] R.Wojcik, S. Majewski, D. Steinbach and A.G. Weisenberger, "High Spatial resolution Gamma Imaging Detector Based on 5" Diameter R3292 Hamamatsu PSPMT," IEEE Trans. Nucl. Sci., vol. 45, no.3, pp.487-491, June, 1998.

[8] Y. Wang, B.M.W. Tsui, and E.C. Frey. "Theoretical analysis of conditions for unique solutions in geometric calibrations for cone-beam and pinhole tomography," submitted to phys. Med. Bio., 2004 